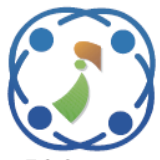

\title{
A Practical Implementation of Memristor Emulator Circuit Based on Operational Transconductance Amplifiers
}

\author{
Apichata Thongrak ${ }^{1 *}$ \\ Suchada Sitjongsataporn ${ }^{2}$ \\ Sanya Khunkhao ${ }^{3}$ \\ Phichet Moungnoul ${ }^{1}$ \\ ${ }^{1}$ Faculty of Engineering, King Mongkut's Institute of Technology Ladkrabang \\ 1 Chalongkrung Rd., Ladkrabang, Bangkok 10520 Thailand \\ ${ }^{2}$ Department of Electronic Engineering, Faculty of Engineering, Mahanakorn University of Technology \\ 140 Cheumsamphan Rd., Nongchok, Bangkok 10530 Thailand \\ ${ }^{3}$ Faculty of Engineering, Rajamangala University of Technology Phra Nakhon \\ 1381 Pracharat 1 Road, Wongsawang Subdistrict, Bang Sue District, Bangkok 10800 \\ * Corresponding author's Email: aphichata.t@rmutp.ac.th
}

\begin{abstract}
As described of electrical circuit have been considering that there are three fundamental passive twoterminal circuit elements; resistor, capacitor, and inductor respectively. In 1971, Prof. L. Chua proposed and described memristor which defines the relationship between magnetic flux and charge. This paper are purposed a memristor emulator circuit based on operational transconductance amplifiers (OTAs). The proposed circuits can be realized using commercially integrated circuits OTA, op amp as AD844, TL084, inductor, capacitor and resistors. The characteristic of memristor emulators can be examined in a practical experiment with the active and passive components. As described, the results could be demonstrated with the memristor circuit application. Furthermore, OTA is used to realize electronically tunable current conveyors emulator circuit. As a result of simulation, the decremental and incremental memristor emulator circuit is suitable for connecting in a series circuit. It was found that the frequency-dependent pinched hysteresis loop of proposed memristor emulator circuits can be updated by changing the value of capacitance and resistance, besides it increases the current gain of current conveyor. In addition to the expected results of memristor $\left(\mathrm{R}_{\mathrm{M}}\right)$, Memcapacitor $\left(\mathrm{C}_{\mathrm{M}}\right)$ and meminductor $\left(\mathrm{L}_{\mathrm{M}}\right)$ are proposed to determine in time-domain characteristics of R-L-C mode circuits. The results of experimental are discussed of phenomena studies by the memory characteristics.
\end{abstract}

Keywords: Memristor, Emulator circuit, Operational transconductance amplifier (OTA).

\section{Introduction}

Recently, the memristors are purposed in form of memory-resistor and memristive devices that were presumed to exist when the link was made to voltagepolarity-dependent. The formulation of a memristor was first devised in 1971 by Chua [1] as a fourth circuit on passive elements such as $\mathrm{R}, \mathrm{L}$ and $\mathrm{C}$ respectively. A number of memristor applications has been rapidly reported for digital logic circuits in [2-4] and neural networks in $[5,6]$.

The memristor emulator circuits based on CMOS differential difference current conveyors
(DDCCs) have been proposed in [7] using memristor emulator circuit using two CFOAs, one OTA, three resistors and two capacitors. A multipleoutput OTA (MO-OTA) has been used to realize either decremental or incremental memristor emulator circuits in [8].

Memristors offer a nonvolatile memory storage with simple device structure as detailed in $[9,10]$ including the artificial neural networks as synaptic weights with a pulse based memristor circuit [2], memristor XOR gate for resistive multiplier [3] and phase shift keying modulators based memristor [4]. 
Table 1. Comparison of the proposed circuit with those of some previous works

\begin{tabular}{|c|c|c|c|}
\hline Circuits & No. of active components & No. of passive components & Decremental/Incremental \\
\hline $\begin{array}{c}\text { Proposed circuit } \\
\text { Fig. 5 }\end{array}$ & $\begin{array}{c}\text { 1-EDDCC } \\
\text { (3-LM13600), } \\
\text { 1-AD633 }\end{array}$ & 2-R, 1-C & Decremental \\
\hline Fig. 6 & $\begin{array}{c}\text { 1-EDDCC } \\
\text { (3-LM13600), } \\
\text { 1-AD633 }\end{array}$ & 2-R, 1-C & Incremental \\
\hline Ref. [16] (2017) & 1-CCTA & 3-R, 1-C, 1-SW & Both \\
\hline Ref. [17] (2017) & 1-DVCCTA & 3-R, 1-C, 1-SW & Both \\
\hline Ref. [18] (2018) & 7-MOS & 1-C & Decremental \\
\hline Ref. [19] (2015) & 3-ECCII (6-LM13600), \\
1-AD633 & 1-R, 1-C & Decremtal \\
\hline
\end{tabular}

Many types of mixed-mode circuits have been studied such as differential voltage current conveyors (DVCCs), second-generation current conveyors (CCIIs), current controlled current conveyors (CCCIIs), DDCCs and OTAs [11]. Based on the light-dependent resistor (LDR), a memcapacitor emulator has been presented using $\mathrm{R}_{\mathrm{M}}-\mathrm{C}_{\mathrm{M}}$ converter and a method is used to satisfy a LDR memristor-based meminductorequivalent circuit detailed in [12].

The modern active devices have been used to realize memristor emulator circuits such as current conveyor transconductance amplifier (CCTA) in [16], differential voltage current conveyor transconductance amplifier (DVCCTA) in [17]. The circuits in [17] provide decremental and incremental memristor emulators into one circuit by selecting the switch. The circuit in [16] is simulated using CMOS implementation of CCTA while the circuit in [17] is simulated using CMOS implementation of DVCCTA whereas experiment tests, these circuits have been built using commercially available ICs AD844 and CA3080. The circuit in [18] realizes memristor emulator circuit based on MOSFET-C.

The comparison of proposed circuits with those previous works are summarized in Table 1. Compared with [16-18], the proposed circuits can be pushed the operating of high frequency by capacitance, resistance and current gain of current conveyor, compared with $[16,18,19]$, the proposed circuits in Figs. 5 and 6 provide decremental and incremental memristor emulators into single circuit and compared with [16, 17], the proposed circuits can be tested both simulation and experiment with the same active and passive components.

In this paper, we introduce a compact circuit model and physical hardware emulation for memristor using the electronically tunable differential different current conveyor (EDDCC). The hardware emulator will demonstrate experimentally memristor dynamics.

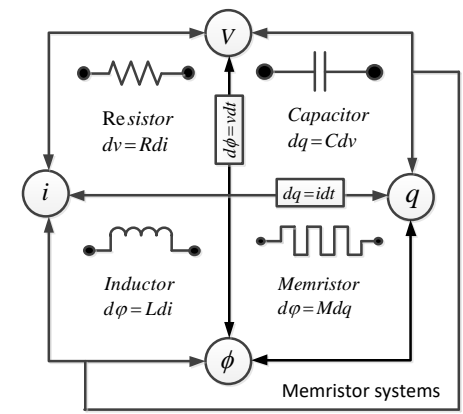

Figure.1 Four fundamental two-terminal circuit elements: resistor, capacitor, inductor and memristor [22]

This paper is organized as follows. The definitions and concept of memristor model is introduced in Section 2 and Section 3 is described about OTA-based ECCII. We present an OTA-based EDDCC memristor emulator that is similar to that of a memristor shown in Section 4. Experimental results and conclusion are given in Section 5 and 6, respectively.

\section{Memristor model}

Following [13], there are four fundamental circuit variables: electric current $i$, voltage $v$, charge $q$ and magnetic flux $\varphi$ described in Fig.1. The memristor, with memristance $M$-provides a functional relation between charge and flux, $d \varphi=M d q$. Basic mathematical definition of a current-controlled memristor for circuit analysis is given by $v=R(w) i$ and $\frac{d w}{d t}(\mathbf{i})$, where $w$ is the state variable and $R$ is a generalized resistance.

The concept of memristive systems is described as $v=R(w, i)$ and $\frac{d w}{d t}(i)$ where $f$ is function of time.

A thin semiconductor film of thickness D sandwiched between two metal contacts is considered in Fig. 2. The total resistance is assigned with 2-variable resistors connected in series, where the resistances are given for the full length $D$ of the 

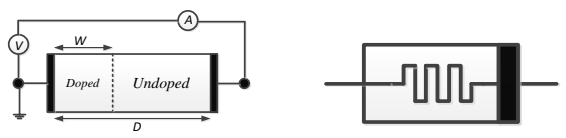

(a)

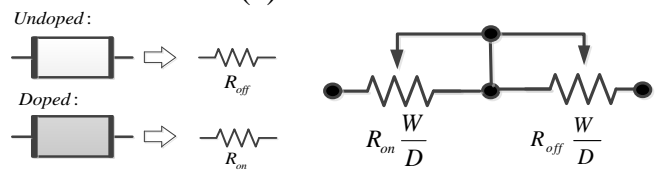

(b)

Figure. 2 Coupled variable-resistor model for a memristor:

(a) structure and symbol of the memristor and (b) diagram with a simplified equivalent circuit

device. However, the semiconductor film has a region with a high concentration of dopants having low resistance $R_{\text {on }}$. The remainder closes to essentially zero dopant concentration with higher resistance $R_{\text {off. An }}$ external bias $v(t)$ across the device will move the boundary between the two regions causing the charged dopants to drift, we get

$$
\begin{gathered}
v(t)=\left\{R_{\text {on }} \frac{w(i)}{D}+R_{o f f}\left(1-\frac{w(i)}{D}\right)\right\} i(t) \\
\frac{d w(t)}{d t}=\mu \frac{R_{o n}}{D} i(t)
\end{gathered}
$$

and $w(t)$ is given as

$$
w(t)=\mu_{v} \frac{R_{o n}}{D} q(t)
$$

By substituting Eq. (3) into Eq. (1), the memristance for $R_{\mathrm{ON}}=R_{\mathrm{OFF}}$ can be expressed as

$$
M(g)=R_{o f f}\left(1-\frac{\mu_{v} R_{o n}}{D^{2}}\right) q(t)
$$

where the term of q-dependent is the crucial support to the memristance. It becomes larger with higher dopant mobilities $\mu_{\mathrm{V}}$ and smaller semiconductor film thicknesses $D$, respectively.

\section{OTA-based electronically tunable second- generation current conveyor (ECCII)}

OTA is important active device that has been used to realize both voltage- and current-mode analog circuits. OTA-based circuits can be tested both simulation and breadboard experiment. Symbol of OTA is shown in Fig.3. The ideal OTA is described by $I_{0}=g_{m}\left(V_{1}-V_{2}\right)$, where is the output current, $g_{m}$ is the transconductance gain, $V_{l}$ and $V_{2}$ denote respectively the non-inverting and inverting input voltages. The transconductance $g_{m}$ of OTA can be controlled using external bias current.

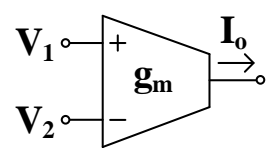

Figure. 3 Circuit symbol of OTA

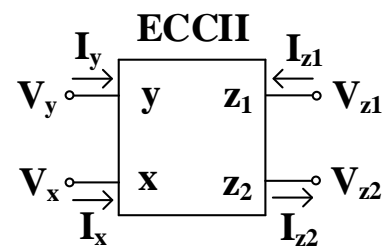

Figure.4 Symbol of ECCII

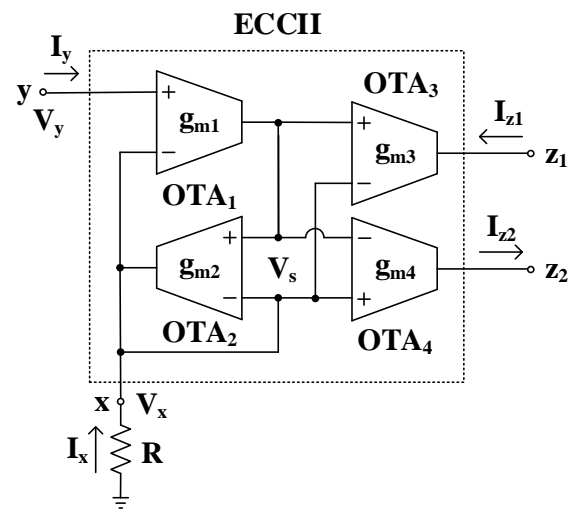

Figure. 5 OTA implementation for ECCII

The symbol of the ECCII is shown in Fig. 4 and its ideal characteristics can be described by

$$
\left[\begin{array}{l}
I_{y} \\
V_{x} \\
I_{z}
\end{array}\right]=\left[\begin{array}{ccc}
0 & 0 & 0 \\
1 & 0 & 0 \\
0 & \mp k & 0
\end{array}\right]\left[\begin{array}{l}
I_{y} \\
V_{x} \\
I_{z}
\end{array}\right]
$$

ECCII has a unity voltage gain between terminals $\mathrm{y}$ and $\mathrm{x}$ and has current gain $\mathrm{k}$ between terminals $\mathrm{z}$ and $\mathrm{z}$. OTA implementation for ECCII can be shown in Fig. 5. Using nodal analysis, the relation between $V_{x}$ and $V_{y}$ can be expressed as

$$
V_{x}=\frac{g_{m 1} g_{m 2} \gamma_{i n} R}{1+g_{m 1} g_{m 2} \gamma_{i n} R} V_{y}
$$

where $g_{m 1}$ and $g_{m 2}$ are respectively the transconductance gains of $\mathrm{OTA}_{1}$ and $\mathrm{OTA}_{2}, \mathrm{R}$ is the given resistor and $\mathrm{rin}$ is the small-signal input resistance of $\mathrm{OTA}_{2}$.

\section{OTA-based electronically tunable differential different current conveyor (EDDCC)}

The part relationship of EDDCC is shown in Fig. 6 which is given as 


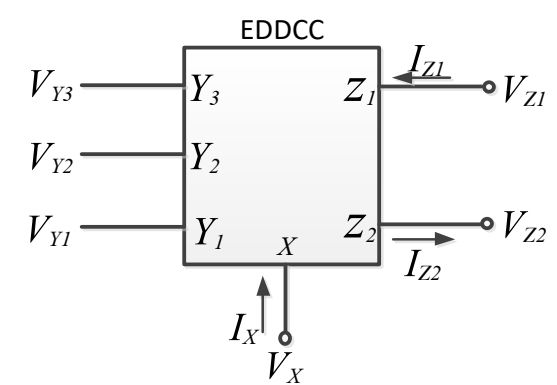

Figure.6 Circuit symbol of EDDCC

$$
\left[\begin{array}{c}
V_{x} \\
I_{y 1} \\
I_{y 2} \\
I_{y 3} \\
I_{z 1} \\
I_{z 2}
\end{array}\right]=\left[\begin{array}{cccc}
1 & -1 & 1 & 0 \\
0 & 0 & 0 & 0 \\
0 & 0 & 0 & 0 \\
0 & 0 & 0 & 0 \\
0 & 0 & 0 & k_{1} \\
0 & 0 & 0 & -k_{2}
\end{array}\right] \cdot\left[\begin{array}{c}
V_{y 1} \\
V_{y 2} \\
V_{y 3} \\
I_{x}
\end{array}\right]
$$

where $g_{m}$ is transconductance of EDDCC.

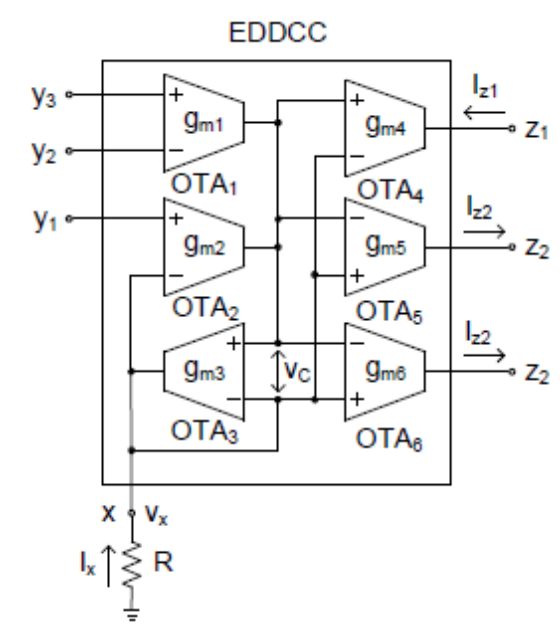

Figure. 7 Symbol of developed EDDCC

OTA implementation for EDDCC based on CMOS is depicted in Fig. 7. The OTA 1 to $\mathrm{OTA}_{3}$ are used to provide a differential difference amplifier (DDA). By using nodal analysis, the relation of voltages $V_{x}, V_{y 1}, V_{y 2}$ and $V_{y 3}$ can be expressed by

$$
V_{y}=\frac{\left(g_{m 2} g_{m 3} \gamma_{i n} R\right) V_{y 1}-\left(g_{m 1} g_{m 3} \gamma_{i n} R\right) V_{y 2}+\left(g_{m 1} g_{m 3} \gamma_{i n} R\right) V_{y 3}}{1+\left(g_{m 3} R+g_{m 2} R+g_{m 3} \gamma_{i n} R\right)}
$$

where $g_{m i}$ is the gain of transconductance of OTA $A_{i}$, $\mathrm{R}$ is the defined resistor and $r_{\text {in }}$ is input resistor in case of small signal of $\mathrm{OTA}_{3}$ which has highly resistance. Relationship between $V x, Y_{1}, Y_{2}$, and $Y_{3}$ Ports for EDDCC is given as

$$
V_{x} \approx V_{y 1}-V_{y 2}+V_{y 3}
$$

From Fig. 7, we assume the current $I_{x}$ is equal to output current of $\mathrm{OTA}_{3}$ which is given as

$$
\begin{gathered}
I_{x}=g_{m 3}\left(V_{C}-V_{x}\right) \\
I_{Z 1}=g_{m 4}\left(V_{C}-V_{x}\right) \\
I_{Z 2}=g_{m 5}\left(V_{x}-V_{C}\right)
\end{gathered}
$$

Substituting this equations

$$
\begin{gathered}
V_{C}-V_{x}=\frac{I_{Z 1}}{g_{m 4}} \\
V_{C}-V_{x}=\frac{I_{Z 2}}{g_{m 5}}
\end{gathered}
$$

into this equation

$$
V_{C}-V_{x}=\frac{I_{x}}{g_{m 3}}
$$

Then, we can rewrite $I_{z 1}$ and $I_{z 2}$ as

$$
\begin{gathered}
I_{Z 1}=\left(\frac{g_{m 4}}{g_{m 3}}\right) I_{x}=k_{1} I_{x} \\
I_{Z 1}=\left(\frac{g_{m 5}}{g_{m 3}}\right) I_{x}=k_{2} I_{x}
\end{gathered}
$$

It is noted that $k_{1}$ and $k_{2}$ are the current gain of EDDCC controlled by $g_{m 4}$ and $g_{m 5}$. Current gain of $I_{z 1}$ and $I_{z 2}$ is amplified by $k_{1}$ and $k_{2}$ that is different from the ordinary DDCC. Hence, the gain $k_{1}$ and $k_{2}$ are independently controlled.

The multi-output EDDCC can simply create by increasing plus-type of EDDCC, when the increased parallel input of OTA is connected to OTA4. If minus-type EDDCC is increased, the parallel input of OTA will increase by connecting with OTA 5 .

Relationship of voltage and current is given as

$$
V(t)=\left\{\left(R_{\text {on }} \frac{w(t)}{D}\right)+R_{\text {off }}\left(1-\frac{w(t)}{D}\right)\right\} i(t)
$$

where $D$ and $W$ are the thick of sandwiched area and doped area in memristor. $R_{o n}$ and $R_{\text {off }}$ are the resistance on the concentrated area of high and low doped. Memristor emulator circuits are proposed in Figs. 8 and 9 with 2-resistor, 1-capacitor and 1analog multiplier which are used the concept followed in [17].

By using the nodal analysis, we get

$$
V_{m}=i_{m} R_{1}+V_{x}
$$




$$
\begin{gathered}
V_{C}=\frac{k_{2}}{R_{1} C_{1}} \int_{0}^{1} v_{m}(\tau) d \tau \\
V_{R}=-k_{1} R_{2}+I_{m}
\end{gathered}
$$

Following the characteristic of input/output of AD844, the voltage $V_{m u l}$ is given as

$$
V_{m u l}=-i_{m} \frac{k_{1} k_{2} R_{2}}{10 R_{1} C_{1}} \int_{0}^{t} v_{m}(\tau) d \tau
$$

Using the input properties of DDCC is given as

$$
\begin{gathered}
V_{x}=V_{y 1}-V_{y 2}+V_{Y 3} \\
V_{m}=i_{m} R_{1}-\left(i_{m} \frac{k_{1} k_{2} R_{2}}{10 R_{1} C_{1}} \int_{0}^{1} V_{m}(\tau) d \tau\right)+V_{s u m}
\end{gathered}
$$

From Eq.(22), we assume $V_{\text {sum }}$ is equal to zero and flux $\phi_{m}(t)$ is defined by

$$
\phi_{m}(t)=\int_{0}^{1} V_{m}(t) d t
$$

Then, Eq. (22) can rewrite as

$$
\frac{V_{m}}{i_{m}}=R_{1}-\left(\frac{k_{1} k_{2} R_{2}}{10 R_{1} C_{1}} \phi_{m}(t)\right)=M(\phi(t))
$$

where $M(\phi(t))$ is the resistance of memristor that is used $k_{1}$ and $k_{2}$ to compensate the attenuation rate constant at 1/10 from multiplier circuit. Moreover, it can compensate the effect from frequency that affect to pinched hysteresis loop behavior of memristor.

From Figs. 8 and 9, the DC voltage $V_{\text {off } 1}, V_{\text {off } 2 \text {, }}$ $V_{\text {off }}$ are the bias current to improve the pinched hysteresis loop behavior, when the characteristic is distorted. Voltage $V_{\text {off } 1}$ and $V_{\text {off }}$ is applied to adjust the symmetry between the positive and negative of pinched hysteresis loop and then $V_{\text {off }}$ is defined to tune the zero-crossing of pinched hysteresis loop.

We assume that $v_{\text {in }}(t)=v_{p} \sin (\omega t)$ is the input of Fig. 8, where $\omega=2 \pi f$ and $V_{p}$ is the amplitude of voltage signal. Then we define

$$
\phi_{m}(t)=-\left(\frac{V_{p}}{\omega}\right) \cos (\omega t)=\left(\frac{V_{p}}{\omega}\right) \cos (\omega-t)
$$

and substitute $\phi_{m}(t)$ in Eq.(30), the memristance is given as

$$
M\left(\phi_{m}(t)\right)=R_{1}-\left(\frac{k_{1} k_{2} R_{2}}{10 R_{1} \omega C_{2}} \cos (\omega t-\pi)\right)
$$

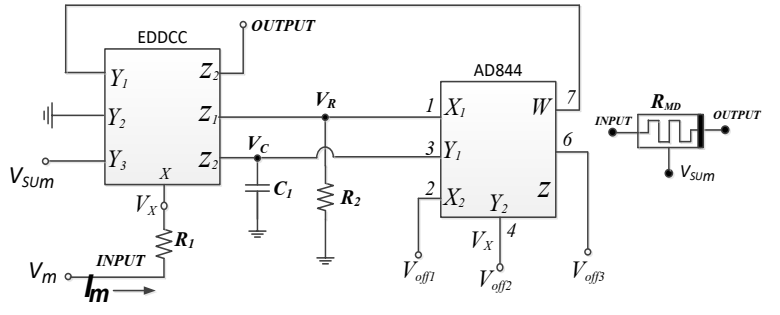

Figure. 8 Emulator circuit symbol of decremental EDDCC memristor and equivalent circuit

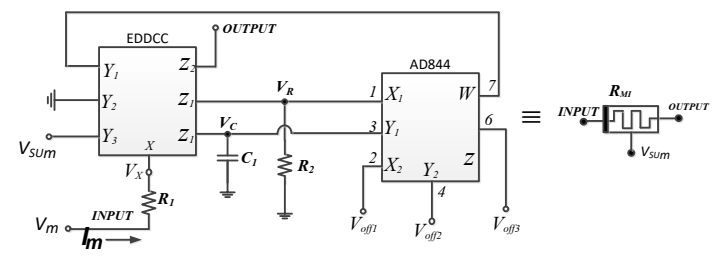

Figure.9 Emulator circuit symbol of incremental EDDCC memristor and equivalent circuit

From Eq.(28), the memristance consists of linear time-variant and linear time-invariant resistors. If the frequency increases, the linear time-invariant resistor will decrease. That mean the dependent frequency is pinched the hysteresis loop, when the frequency is increased. At the fix time, the linear time-variant resistor is updated by frequency that will update this circuit by adjusting $\mathrm{k}_{1}$ and $\mathrm{k}_{2}$, respectively.

\section{Simulation results}

In order to investigate the operation of the proposed memristor emulator circuits, the circuits of Figs. 7 to 9 were simulated using PSPICE simulations. ECCII in Fig. 3 and EDDCC in Fig. 5 were implemented using commercially available IC LM13600N [57]. A commercially available IC AD844J [56] was used for voltage multiplier.

Table 2. Summarized performances of ECCII and EDDCC

\begin{tabular}{|l|c|}
\hline \multicolumn{1}{|c|}{ Parameters } & Value \\
\hline Supply voltage & $\pm 10 \mathrm{~V}$ \\
\hline OTA & LM13600N \\
\hline $\mathrm{V}_{\mathrm{x}} / \mathrm{V}_{\mathrm{y}}$ and $\mathrm{V}_{\mathrm{x}} / \mathrm{V}_{\mathrm{y} 1}$ (no load) & $-0.9 \mathrm{~V}$ to $0.9 \mathrm{~V}$ \\
\hline $\mathrm{V}_{\mathrm{x}} / \mathrm{V}_{\mathrm{y} 2}$ and $\mathrm{V}_{\mathrm{x}} / \mathrm{V}_{\mathrm{y} 3}($ no load) & $-0.1 \mathrm{~V}$ to $0.1 \mathrm{~V}$ \\
\hline$-3 \mathrm{~dB}$ bandwidth $\left(\mathrm{V}_{\mathrm{x}} / \mathrm{V}_{\mathrm{yi}}\right) ; \mathrm{i}=1,2,3$ & $7.3 \mathrm{MHz}$ \\
\hline$-3 \mathrm{~dB}$ bandwidth $\left(\mathrm{I}_{z} / \mathrm{I}_{\mathrm{x}}\right)$ & $11 \mathrm{MHz}$ \\
\hline $\mathrm{R}_{\mathrm{yi}}: \mathrm{C}_{\mathrm{y}} ; \mathrm{i}=1,2,3$ & $185 \mathrm{k} \Omega: 8.4 \mathrm{pF}$ \\
\hline $\mathrm{R}_{\mathrm{x}}: \mathrm{L}_{\mathrm{x}}$ & $17 \Omega: 24 \mu \mathrm{H}$ \\
\hline $\mathrm{R}_{\mathrm{z}}: \mathrm{C}_{\mathrm{z}}$ & $22 \mathrm{k} \Omega: 6.12 \mathrm{pF}$ \\
\hline
\end{tabular}




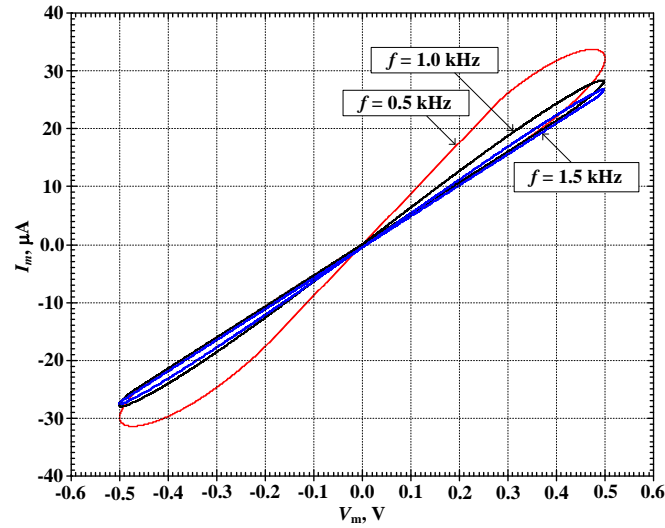

Figure.10 Simulated frequency dependent pinched hysteresis loop of memristor emulator at current gain $\mathrm{k}_{1}=$ $\mathrm{k}_{2}=1$ and different frequencies

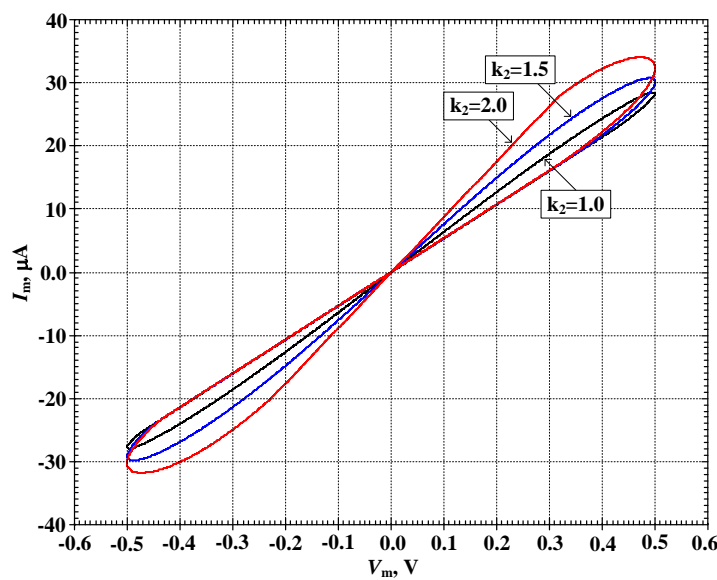

Figure.11 Simulated frequency dependent pinched hysteresis loop of memristor emulator at $f=1 \mathrm{kHz}$ and $\mathrm{k}_{1}$ $=1$ with different current gains $\mathrm{k}_{2}$

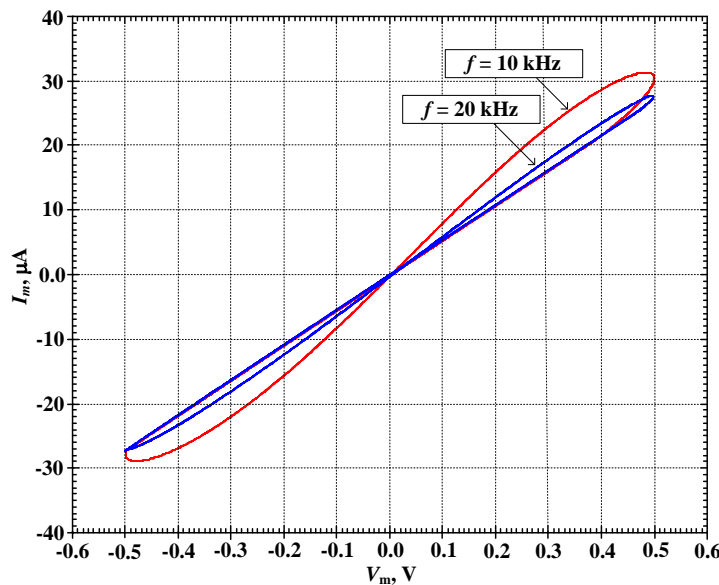

Figure.12 Simulated frequency dependent pinched hysteresis loop of memristor emulator at $\mathrm{k}_{1}=1$ and $\mathrm{k}_{2}=$ 20 with frequency of 10 and $20 \mathrm{kHz}$

The commercially available IC LM13600N was supplied by the DC supply voltages of $\pm 10 \mathrm{~V}$ while $\mathrm{OTA}_{1}, \mathrm{OTA}_{2}$ for ECCII in Fig. 3 and OTA , OTA $_{2}$, $\mathrm{OTA}_{3}$ for EDDCC in Fig. 5 were biased by the DC current sources of $50 \mu \mathrm{A}$. The simulated performances of the ECCII and EDDCC were shown in Table 2.

The proposed decremental memristor emulator circuits in Figs. 8 and 9 were simulated. The values of resistances and capacitance were given as $R_{l}=15$ $\mathrm{k} \Omega, R_{2}=5 \mathrm{k} \Omega$ and $C_{l}=1 \mathrm{nF}$. In case to simulate the proposed incremental memristor emulator circuits in Fig.8, the low resistance value such as $R_{l}=5 \mathrm{k} \Omega$ should be used. The DC voltages $V_{\text {off } 1}, V_{\text {off } 2}$, and $V_{\text {off } 3}$ in Fig.9 were given as $26 \mathrm{mV}, 0 \mathrm{~V}$ and $-50 \mathrm{mV}$, respectively, and current gain $k_{l}$ was fixed as $1\left(k_{l}=\right.$ 1).

Fig. 10 shows the simulated frequencydependent pinched hysteresis loop in voltage-current relationship corresponding to $v_{m}(t)$ versus $i_{m}(t)$ plane for the frequencies of $0.5,1.0$ and $1.5 \mathrm{kHz}$ with amplitude of applied signal $0.5 \mathrm{~V}$ (peak) constant and the current gain $\mathrm{k}_{2}$ was 1 . This result was confirmed that when amplitude of applied signal and capacitance-value constants, the proposed memristir emulator circuit depends on the frequency of the exciting source.

Fig. 11 shows the simulated frequencydependent pinched hysteresis loop in voltage-current relationship corresponding to $v_{m}(t)$ versus $i_{m}(t)$ plane for the current gains $\mathrm{k}_{2}$ of $1.0,1.5$ and 2.0 while the frequency of $1 \mathrm{kHz}$ with amplitude of applied signal $0.5 \mathrm{~V}$ (peak) constants. This result was confirmed that the frequency-dependent pinched hysteresis loop in voltage-current relationship of the proposed circuits can also be controlled by adjusting current gains of current conveyor.

To confirm Eq. (17) that by increasing the current gain of current conveyor, the pinched hysteresis loop behavior of proposed memristor emulator circuit can be pushed for operating at higher frequency. Fig. 12 shows the simulated frequency-dependent pinched hysteresis loop in voltage-current relationship corresponding to $v_{m}(t)$ versus $i_{m}(t)$ plane for the current gain $k_{2}=20$. In this case, the frequencies of the exciting source of 10 $\mathrm{kHz}$ and $20 \mathrm{kHz}$ at amplitude of $0.5 \mathrm{~V}$ (peak) were applied and value of capacitance $C_{1}$ is constant. In this result, the voltages $V_{\text {off1 }}=48 \mathrm{mV}$ and $V_{\text {off3 }}=-$ $40 \mathrm{mV}$ were used to obtain perfect the pinched hysteresis loop behavior.

The proposed decremental memristor emulator circuit in Fig. 8 was and simulated. The values of resistances and capacitance were similar to the simulation in Fig. 8 The circuit was design as $\mathrm{k}_{1}=\mathrm{k}_{2}$ $=1$. Fig. 13 shows simulated frequency-dependent 


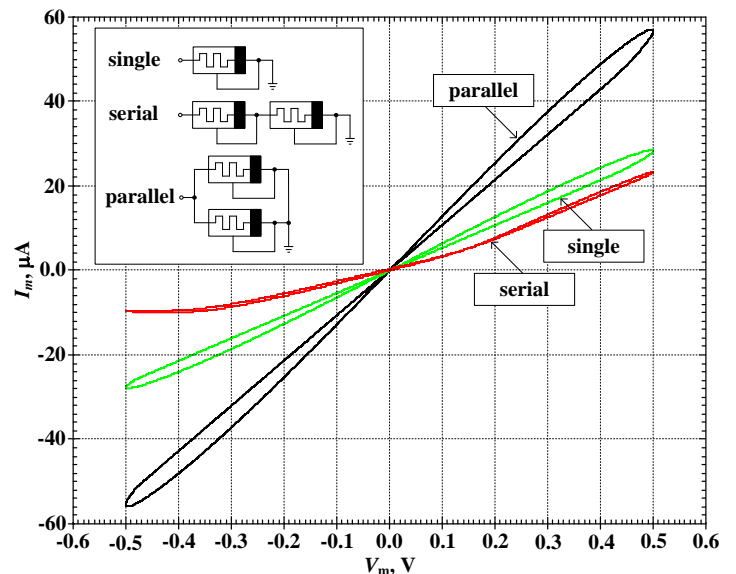

Figure.13 Simulated pinched hysteresis loop of memristor at $1 \mathrm{kHz}$ frequency with series and parallel connection

pinched hysteresis loop in voltage-current relationship corresponding to $v_{m}(t)$ versus $i_{m}(t)$ plane for the frequency of $1 \mathrm{kHz}$ with amplitude of applied signal $0.5 \mathrm{~V}$ (peak) constant. This result was confirmed that proposed circuit in Fig. 9 can be applied to connection in serial and in parallel.

\section{Circuit implementation of $\mathbf{R}_{M}-\mathbf{C}_{M}$ and $\mathbf{R}_{M^{-}}$ $L_{M}$ convertor}

Following [14], the memcapacitor and meminductor emulators are constructed using AD844 shown in $\mathrm{R}_{\mathrm{M}}-\mathrm{C}_{\mathrm{M}}$ convertor in Fig. 14. Relationship of $v_{C M}$ and $v_{R M}$ is give as $v_{R M}=v_{C M}$. Then, OTA formed by AD844 to produce the current $i_{R M}$ is given as

$$
i R_{M}=\left(\frac{1}{R C}\right) \int i C_{M} d t
$$

Memcapacitance from $\mathrm{R}_{M}-\mathrm{C}_{\mathrm{M}}$ is defined as

$$
C_{M}=\frac{d \sigma C_{M}}{d \phi C_{M}}=\frac{R C}{R_{M}}
$$

Fig. 15 depicts the $\mathrm{R}_{\mathrm{M}}-\mathrm{L}_{\mathrm{M}}$ convertor, when $v_{X}=x_{Y}$, $v_{Z}=v_{\text {output }}, i_{Z}=i_{X}$ that is used for $\mathrm{R}_{\mathrm{M}}-\mathrm{L}_{\mathrm{M}}$ convertor. The current through the equivalent meminductor $i_{L M}$ is defined as

$$
i_{L M}=\frac{\left(0-V_{x}\right)}{R_{1}}, \quad i_{R M}=\frac{\left(0-V_{y}\right)}{R_{3}}
$$

Then, the relationship of $v_{L M}$ and $v_{R M}$ as

$$
\begin{aligned}
& v_{L M}=v_{z}=v_{y^{\prime}}=v_{x \prime} \\
& v_{R M}=\frac{1}{R_{2} C_{2}} \int v_{L M} d t
\end{aligned}
$$

Therefore, the meminductance is obtained from $\mathrm{R}_{\mathrm{M}^{-}}$ $\mathrm{L}_{\mathrm{M}}$ converter as

$$
L_{M}=\frac{d \sigma C_{M}}{d q L_{M}}=\frac{R_{1} R_{2} R_{3}}{R_{1} R_{M}}
$$

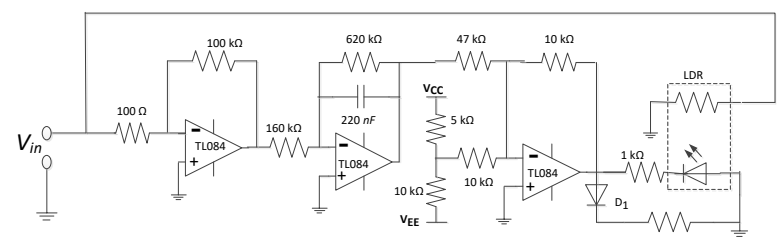

Figure.14 Schematic of proposed $\mathrm{R}_{\mathrm{M}}-\mathrm{C}_{\mathrm{M}}$ circuit

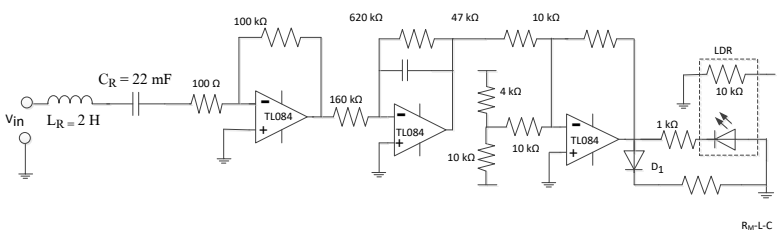

Figure.15 Schematic of proposed $\mathrm{R}_{\mathrm{M}} \mathrm{L}-\mathrm{C}$ circuit

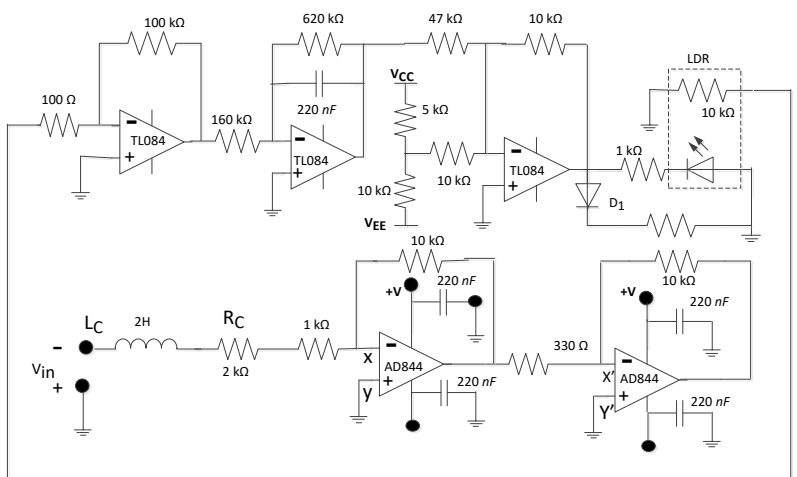

Figure.16 Schematic of proposed R-L- $\mathrm{C}_{\mathrm{M}}$ circuit

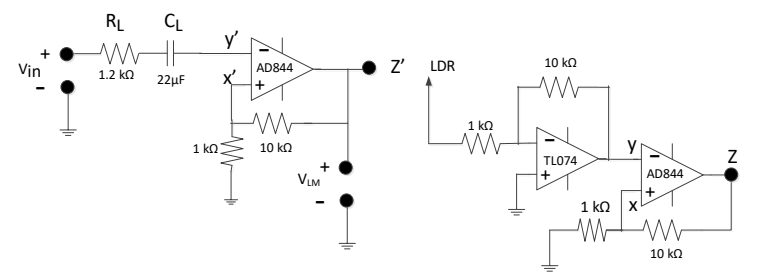

Figure. 17 Schematic of proposed $\mathrm{R}-\mathrm{L}_{\mathrm{M}}-\mathrm{C}$ circuit

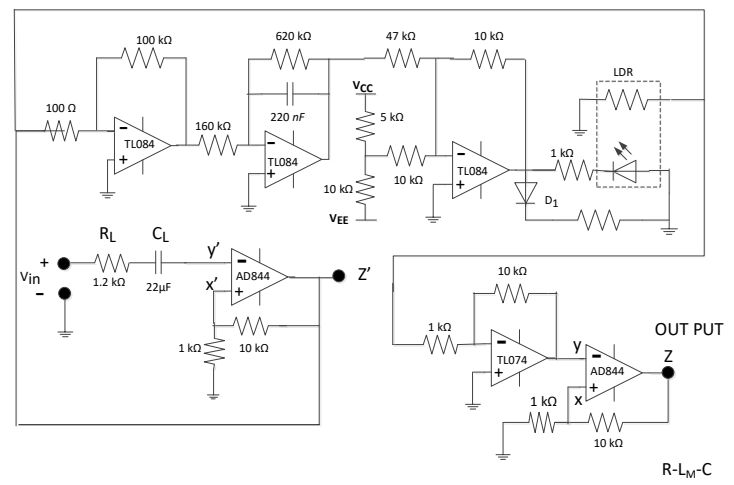

Figure.18 Schematic of proposed R- $\mathrm{L}_{\mathrm{M}}-\mathrm{C}$ circuit 


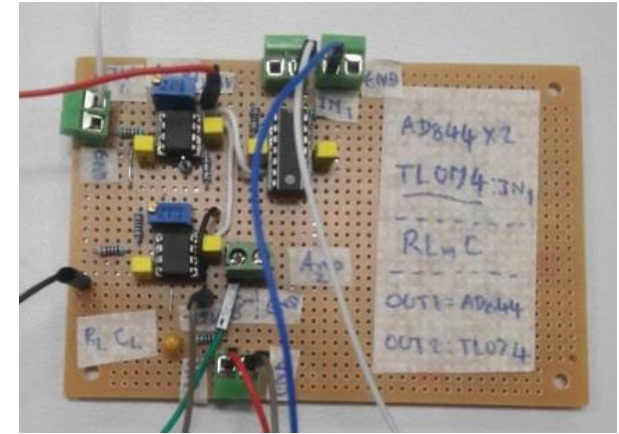

Figure.19 Physical implementation of R- $\mathrm{L}_{\mathrm{M}}-\mathrm{C}$ circuit from schematic shown in Fig. 18

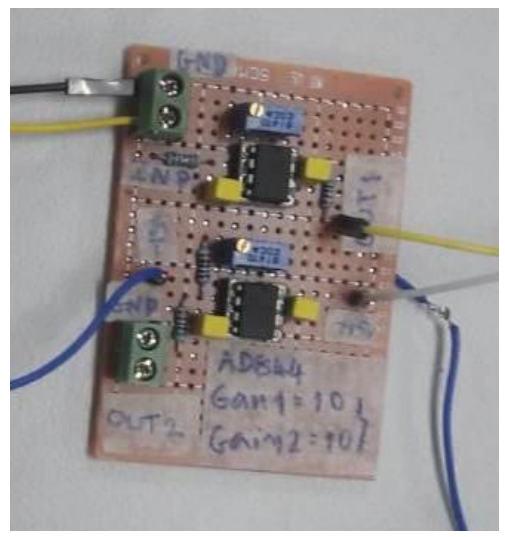

Figure.20 Physical implementation of R-L- $\mathrm{C}_{M}$ circuit is used schematic of proposed $\mathrm{R}_{\mathrm{M}}-\mathrm{C}_{\mathrm{M}}$ circuit in Fig.14

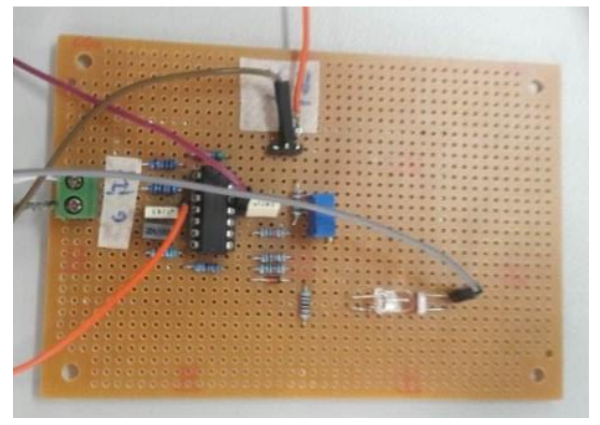

Figure.21 Experimental setup of LDR circuit is used schematic of proposed R-L- $\mathrm{C}_{M}$ circuit in Fig. 16

\section{Experimental results for characteristics of $\mathbf{R}_{M}-\mathrm{L}-\mathrm{C}$ and $\mathrm{R}-\mathrm{L}_{M}-\mathrm{C}$ circuits}

We consider the design of equivalent circuit for RLC-mode circuit in the $\mathrm{R}_{\mathrm{M}}-\mathrm{L}-\mathrm{C}$ and $\mathrm{R}-\mathrm{L}_{\mathrm{M}}-\mathrm{C}$ circuits shown in Figs. 17-18, respectively. Experimental setup for LDR circuit used is depicted in Fig. 21.

\subsection{Experiment of $R_{M-L-C}$ circuit}

Design of $R_{M-L-C}$ circuit using LDR-based memristor-equivalent circuit shown in Fig. 15.

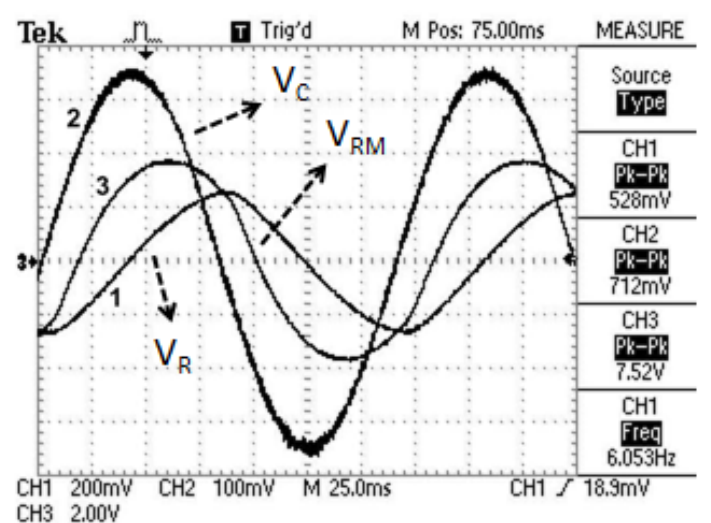

Figure.22 Experimental result $\mathrm{v}_{\mathrm{RM}}, \mathrm{v}_{\mathrm{CR}}, \mathrm{v}_{\mathrm{LR}}$ of $R_{M}-L-C$ circuit at $f=6 \mathrm{~Hz}, \mathrm{v}_{\text {in }}=2 \mathrm{~V}$

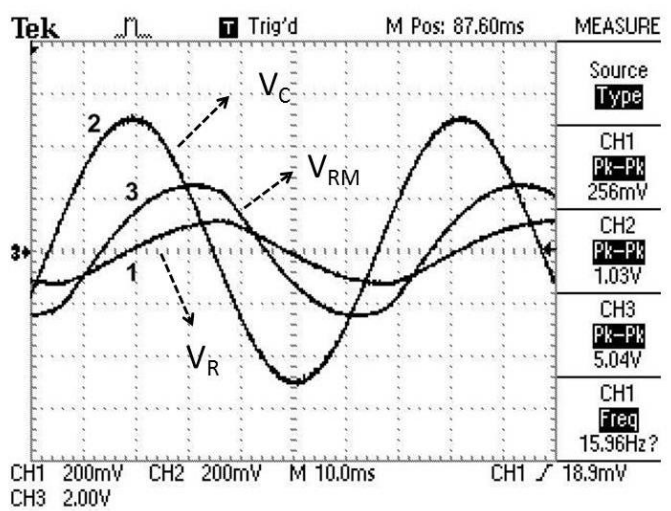

Figure.23 Experimental result $v_{R M}, v_{C R}, v_{L R}$ of $R_{M}-L-C_{M}$ circuit at $f=16 \mathrm{~Hz}, v_{\text {in }}=2 \mathrm{~V}$

From the experiment setup of $R_{M}-L-C_{M}$ circuit are performed at $6 \mathrm{~Hz}$ and $16 \mathrm{~Hz}$ for $v_{\text {in }} 2 \mathrm{~V}$ sinusoidal signal of input voltage shown in Figs. 22 and 23. The voltage $v_{C R}$ across the capacitor $C_{R}$, voltage $v_{L R}$ across the capacitor $L_{R}$, and voltage $v_{R M}$ across the memristor for the given frequencies can be seen that $v_{R M}$ is ahead of $v_{C R}$ and $v_{R M}$ lags behind $v_{L R}$. These results demonstrate that LDR-memristor equivalent circuit can be occupied both the memristor circuit and memory-less resistor characteristics.

\subsection{Experiment of $R-L_{M}-C$ circuit}

Design of $R-L_{M} C$ circuit using LDR-based memristor-equivalent circuit is shown in Fig. 17.

The experiment setup of $R-L_{M}-C$ circuit are performed at $16 \mathrm{~Hz}$ and $30 \mathrm{~Hz}$ for $2 \mathrm{~V}$ sinusoidal signal of input voltage shown in Figs. 24 and 25. The voltage $v_{C R}$, voltage $v_{L R}$, and voltage $v_{R M}$ for the given frequencies can be realized that $v_{R M}$ is ahead of $v_{C R}$ and $v_{R M}$ lags behind $v_{L R}$ 


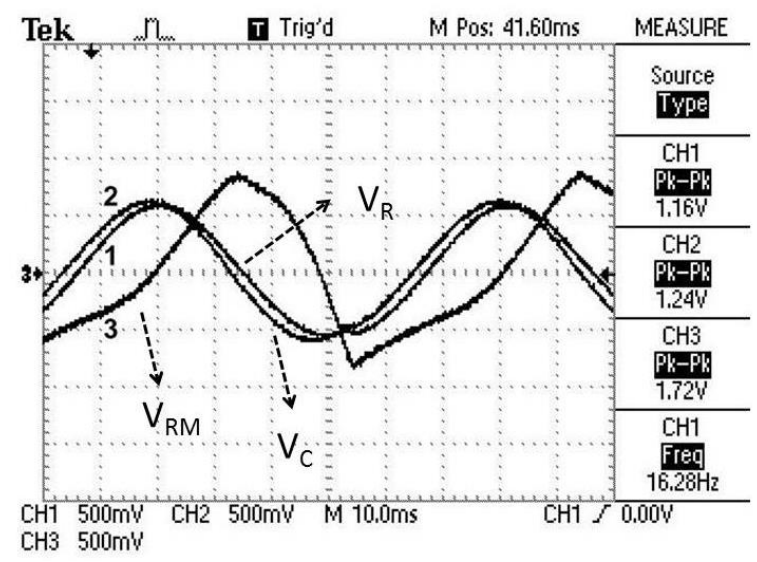

Figure.24 Experimental result $\mathrm{v}_{\mathrm{RM}}, \mathrm{v}_{\mathrm{CR}}, \mathrm{v}_{\mathrm{LR}}$ of $R-L_{M^{-}} C$ circuit at $f=16 \mathrm{~Hz}, v_{\text {in }}=2 \mathrm{~V}$

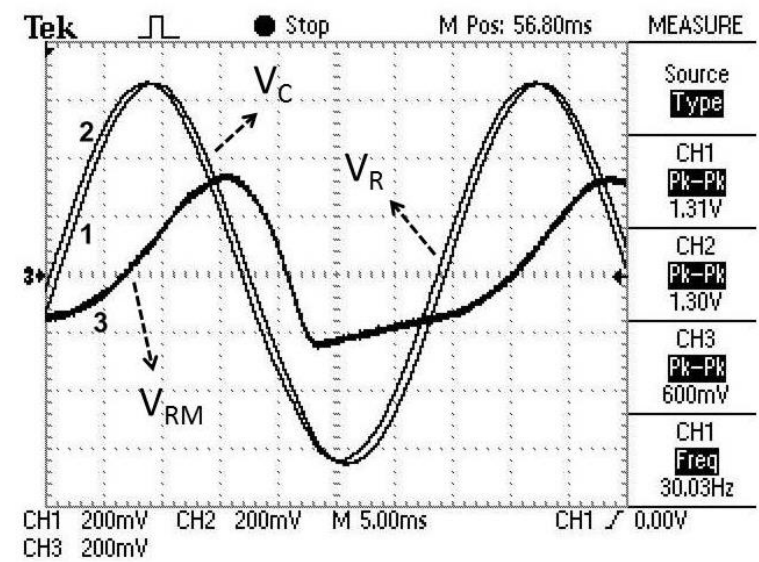

Figure.25 Experimental result $v_{R M}, v_{C R}, v_{L R}$ of $R-L_{M^{-}} C$ circuit at $f=30 \mathrm{~Hz}, v_{i n}=2 \mathrm{~V}$

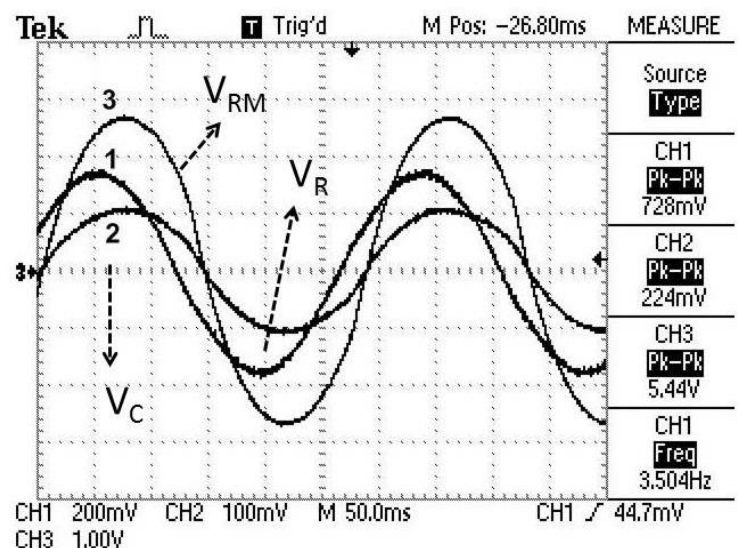

Figure. 26 Experimental result $v_{R M} v_{C R}, v_{L R}$ of $R-L_{M}-C$ circuit at $f=3.5 \mathrm{~Hz}, v_{\text {in }}=2 \mathrm{~V}$

\subsection{Experiment of $R-L-C_{M}$ circuit}

Design of $R-L-C_{M}$ circuit using LDR-based memristor-equivalent circuit is shown in Fig. 18.

The memory characteristics of the memristor $\mathrm{V}_{\mathrm{RM}}$ are not normal sinusodial waveform shown in Figs. 26 and 27. This mechanism are mainly of non-

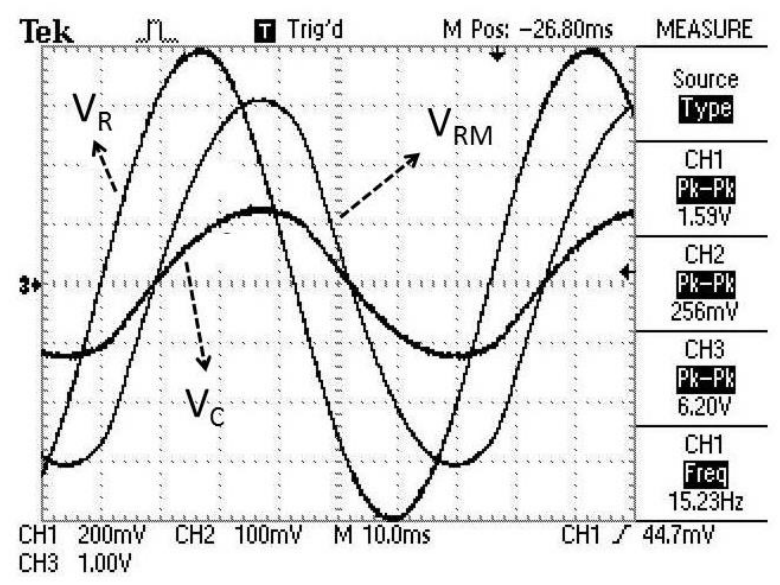

Figure. 27 Experimental result $v_{R M}, v_{C R}, v_{L R}$ of $R-L_{M^{-}} C$ circuit at $f=15 \mathrm{~Hz}, v_{i n}=2 \mathrm{~V}$

linear characteristics of memristor. Futhermore, the measurement phase relation is obtained at low frequency of sinusoidal, which is applied to a memristor device. After that the frequency are increases, then the phase shift is very narrow until it becomes as a line.

\section{Conclusion}

We have proposed the memristor emulator circuits based on commercially available ICs as AD844, TL074, TL084, resistors, inductors and capacitors. OTA has been used to realize ECCII and EDDCC, which are the important active element. Proposed emulator circuits with memresitiveelement-equivalent circuits have been simulated by PSPICE simulator and circuit board is used for experiment tests. RLC-mode circuits are studied including with memristor, memcapacitor and meminductor. In the practical experiments, the proposed memcapacitor and meminductor circuits based on the LDR memristor-equivalent circuit can be verified that the experimental results are agreed with the proposed theory.

\section{Acknowledgments}

This work was supported by Rajamangala University of Technology Phra Nakhon.

\section{References}

[1] L. O. Chua, "Memristor-The Missing Circuit element", IEEE Transactions Circuit Theory, Vol. CT-18, pp. 507-511, 1971.

[2] H. Kim, M. P. Sah, C. Yang, T. Roska, and L. O. Chua, "Neural Synaptic Weighting with a Pulse-based Memristor Circuit", IEEE Transactions on Circuits and System-I: Regular Papers, Vol. 59, pp. 148-158, 2012. 
[3] S. Shin, K. Kim, and S.M. Kang, "Memistor XOR for Resistive Multiplier", Electronics Letters, Vol. 48, pp. 78-80, 2012.

[4] I. C. Goknar, F. Oncul, and E. Minayi, "New Memristor Applications: AM, ASK, FSK, and BPSK Modulators", IEEE Antennas and Propagation Magazine, Vol. 55, pp. 304-313, 2013.

[5] X. Y. Wang, A. L. Fitch, H. H. C. Lu, and W. G. Qi, "Design of a Memcapacitor Emulator based on a Memristor", Physics Letters A, Vol. 376, pp. 394-399, 2012.

[6] D. Yu, H. H. C Iu, A. L. Fitch, and L. Yan, "A Floating Memristor Emulator based Relaxation Oscillator", IEEE Transactions on Circuits and Systems- I: Regular Papers, pp. 2888-2896, 2014.

[7] T. Abuelma'atti and Z. I. Khalifa, "A Continuous-Level Memristor Emulator and Its Application in a Multivibrator Circuit", International Journal of Electronics and Communications, Vol. 69, pp. 771-775, 2015.

[8] Y. Babacan, A. Yesil, and F. Kacar, "Memristor Emulator with Tunable Characteristic and Its Experimental Results", International Journal of Electronics and Communications, Vol. 81, pp. 99-104, 2017.

[9] S. Shin, K. Kim, and S. M. Kang, "Memristor Applications for Programmable Analog ICs", IEEE Transactions on Nanotechnology, Vol. 10, pp. 266-274, 2011.

[10] W. D. Jemison, "Variable Gain Amplifier Circuit using Titanium Dioxide Memristors", IET Circuit Devices and System, pp. 59-65, 2011.

[11] C. N. Lee, "Multiple-mode OTA-C Universal Biquad Filters", Circuits System Signal Processing, Vol. 29, pp. 263-274, 2010.

[12] X. Y. Wang, A. L. Fitch, H. H. C. Iu, V. Sreeram, and W. G. Qi, "Design of a Memcapacitor Emulator based on Memristor", Physic Letter A, Vol. 396, pp. 394-399, 2012.

[13] D. B. Strukov, G. S. Snider, D. R. Stewart, and R. S. Williams, "The Missing Memristor Found", Nature, Vol. 453, pp. 80-83, 2008.

[14] H. Kim, M. P. Sah, Y. Changju, S. Cho, and L. O Chua, "Memristor Emulator for Memristor Circuit Applications", IEEE Transactions on Circuits and Systems I: Regular Papers, pp. 2422-2431, 2012.

[15] Y. Liang, H. Chen, and D. S. Yu, "A Practical Implementation of a Floating Memristor-less Meminductor Emulator", IEEE Transactions on Circuits and System-II: Express Briefs, Vol. 61, pp. 299-303, 2014.
[16] R. K. Ranjan, N. Rani, R.Pal, S. K. Paul, and G. Kanyal, "Single CCTA based High Frequency Floating and Grounded Type of Incremental/Decremental Memristor Emulator and Its Application", Microelectronics Journal, Vol. 60, pp. 119-128, 2017.

[17] R. K. Ranjan, N. Raj, N. Bhuwal, and F. Khateb, "Single DVCCTA based High Frequency Incremental/Decremental Memristor Emulator and Its Application", International Journal of Electronics and Communications, Vol. 82, pp. 177-190, 2017.

[18] A. Yesil, "A New Grounded Memristor Emulator based on MOSFET-C", International Journal of Electronics and Communications, Vol. 91, pp. 143-149, 2018.

[19] M. Kumngern, "A Floating Memristor Emulator Circuit using Operational Transconductance Amplifiers", In: Proc. of IEEE Conference on Electron Devices and Solid-State Circuits, 2015. 